\title{
Bispecific antibody directs T cells to solid tumours
}

ERY974 ...
is one of a
growing list
of bispecific
antibodies
that target
novel antigens
with tumour-
restricted
expression
patterns

Using T cells to attack solid tumours is an attractive option for the treatment of cancer, but requires the presence of a neoantigen on the tumour to engage the T cell. A new paper from Chugai Pharmaceutical uses a bispecific antibody, ERY974, to direct $\mathrm{T}$ cells to non-immunogenic tumours using expression of glypican 3 (GPC3) to confer tumour specificity. Treatment with ERY974 reduced tumour volume in mice with various xenograft tumours including lung, liver and ovary.

Blinatumomab, a bispecific antibody that engages CD3 on T cells and CD19 on B cell tumours, has recently

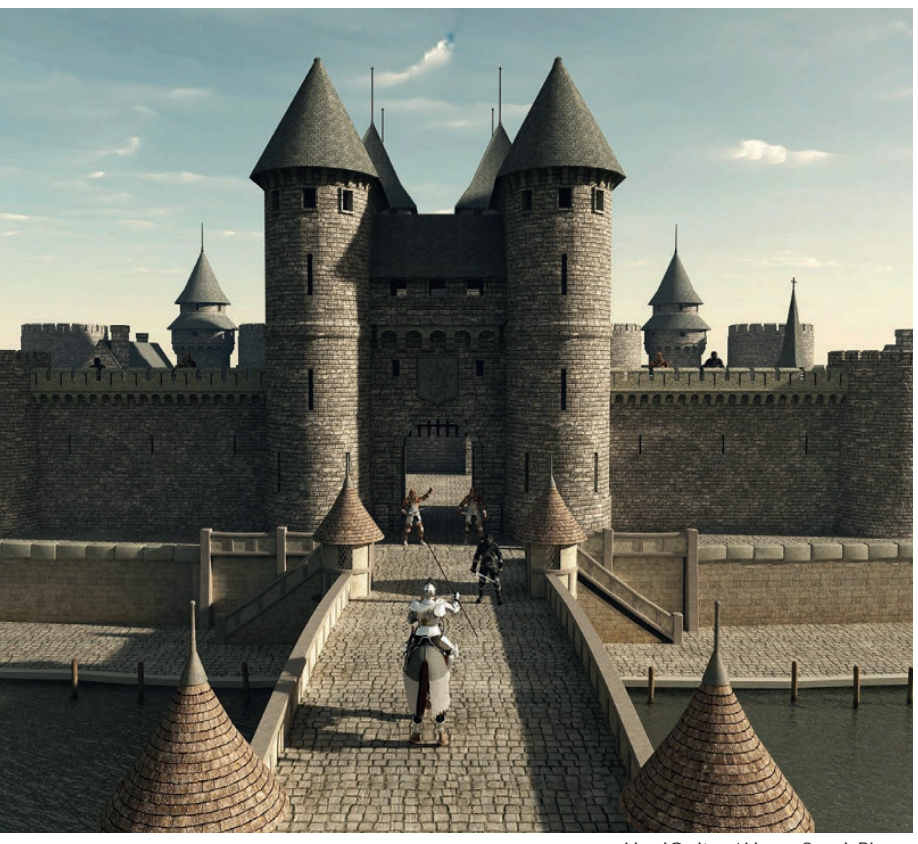

AlgolOnline/Alamy Stock Photo been approved by the FDA for the treatment of a subset of patients with acute lymphoblastic leukaemia. Numerous other bispecific antibodies that target haematological malignancies are in clinical trials. In the new paper, the authors sought to harness bispecific antibodies that engage CD3 to direct $\mathrm{T}$ cells to a target antigen on solid tumours. To reduce the likelihood of on-target, off-tumour toxicity, they looked for proteins expressed almost exclusively in tumours. "Not many proteins were found that met such strict criteria," explains corresponding author Junichi Nezu. "We searched for a target in a comprehensive way by looking at mRNA expression data and conducting protein level examination by immunohistochemistry," says Nezu. The researchers settled on GPC3.

Nezu and his team chose an immunoglobulin G-like format for two reasons: to enable an administration schedule comparable to that of general antibody drugs and to ensure robust manufacturability. They then investigated the potency of ERY974 in GPC3-positive cell lines and in xenograft models that were injected with human T cells. Most of these tumours shrunk from their original size. Similarly, in a human CD3 transgenic mouse model innoculated with human GPC3-expressing mouse tumours, treatment with ERY974 shrunk the tumour in a GPC3-dependent manner.
The clinical experience with immune-activating therapies, such as chimeric antigen receptor (CAR) $\mathrm{T}$ cells, suggests that such therapies can cause potentially lethal cytokine release, which must be controlled with corticosteroid premedication. Indeed, treatment of cynomolgus monkeys with ERY974 induced a transient increase in blood cytokine levels, suggesting that corticosteroid premedication might be needed in patients. Encouragingly, pretreatment with corticosteroids did not affect the antitumour efficacy of ERY974 in mouse models.

ERY974, which is currently in a phase I trial in patients with advanced solid tumours (ClinicalTrials.gov identifier: NCT02748837), is one of a growing list of bispecific antibodies that target novel antigens with tumour-restricted expression patterns, including carcinoembryonic antigen, trophoblast glycoprotein and epithelial cell adhesion molecule. Other GPC3-based treatments are also in clinical development: Chugai has a GPC3 antibody in a phase II trial for hepatocellular carcinoma and multiple institutes are developing CAR T cell therapies that recognise GPC3.

Megan Cully

ORIGINAL ARTICLE Ishiguro, T. et al. An anti-glypican $3 / C D 3$ bispecific T cell-redirecting antibody for treatment of solid tumors. Sci. Transl Med. 9, eaal4291 (2017) 